Editorial

\title{
Flow and Heat or Mass Transfer in the Chemical Process Industry
}

\author{
Dimitrios V. Papavassiliou * (D) and Quoc Nguyen \\ School of Chemical, Biological and Materials Engineering, The University of Oklahoma, Norman, OK 73019, \\ USA; quocnguyen@ou.edu \\ * Correspondence: dvpapava@ou.edu; Tel.: +1-405-325-5811
}

Received: 24 August 2018; Accepted: 28 August 2018; Published: 28 August 2018

Keywords: convection; diffusion; reactive flows; two-phase flow; computational fluid mechanics

Flow through processing equipment in a chemical or manufacturing plant (e.g., heat exchangers, reactors, separation units, pumps, pipes, etc.) is coupled with heat and/or mass transfer. Rigorous investigation of this coupling is important for equipment design. Generalizations and empiricisms served practical needs in prior decades; however, such empiricisms can now be revised or altogether replaced by understanding the interplay between flow and transfer. Currently available experimental and computational techniques can make this possible. Typical examples of the importance of flow in enhancing the transfer of heat and mass is the contribution of coherent flow structures in turbulent boundary layers, which are responsible for turbulent transfer and mixing in a heat exchanger, and the contribution of swirling and vortex flows in mixing. Furthermore, flow patterns that are a function of the configuration of a porous medium are responsible for transfer in a fixed-bed reactor or a fluid-bed regenerator unit.

The goal of this special issue is to provide a forum for recent developments in theory, state-of-the-art experiments, and computations on the interaction between flow and transfer in single and multi-phase flow, and from small scales to large scales, as they are important for the design of industrial processes. It includes papers that cover applications in biological fluid mechanics, microfluidics, membranes, turbulent flows, and gas-liquid flows.

In microfluidics, Kanaris and Mouza [1] proposed a new design for a micromixer based on the insertion of helical structures into a straight tube. Mixing is induced by creating a swirling flow. This design is different from current devices that are based on microtubes, which are themselves helical or twisting. A detailed computational fluid-dynamics study of the proposed design led to the development of model equations for the prediction of mixing efficiency and pressure drop in the microfluidic mixer.

In bio-applications, Williams et al. [2] explored the use of perfusion bioreactors for bone-tissue engineering. In order to monitor the process of cell proliferation for stem cells seeded on polymeric scaffolds, they combined experiments with simulations. Intermittent samples were taken over a period of 16 days from the bioreactor and imaged with tomographic techniques. Flow simulations conducted on flow domains generated by exactly these images followed, allowing the calculation of the flow-induced stresses on the scaffolds. This process led to the generation of data on the average shear stress vs. reaction time- data that are critical for the thoughtful design of tissue engineering scaffolds. In the second bio-application paper, Passos et al. [3] investigated the delivery of drugs through dentinal tissue. After validation with sophisticated experiments that use micro-laser-induced fluorescence ( $\mu$-LIF), computational studies followed to characterize the details of the diffusion of the drugs through the tissue, and to develop a model with appropriate design parameters. Both the Williams et al. [2] and the Passos et al. [3] papers deal with flows and transport in microfluidic environments. 
In large-scale applications with industrial interest, Sobhansrbandi et al. [4] investigated methane reformation with cold plasma. The conversion of methane to syngas (hydrogen and carbon monoxide) was found to be economically feasible, taking advantage of the vortex flow created by a smart design of the reformer. Computational fluid dynamics revealed that the vortex flow enhances the mixing of the reactant gases and the rate of hydrogen production. In the same area of thermal fluids, Duong et al. [5] presented the process of designing eco-friendly cellulose fibers from paper waste. Recycling paper, as described in this work, produced aerogels for thermal insulation that performed as well or better than commercially available insulation materials. The third paper with direct industrial relevance is a detailed analysis of the behavior of the spiral-wound membrane-a separation method used in reverse osmosis and desalination processes for water treatment. Koutsou et al. [6] used three-dimensional (3D) direct numerical simulations to show how flow and mass transfer are affected by the membrane fouling process. Correlations between the friction factor and the mass transfer coefficient obtained through this study have practical use and can also be employed to develop realistic dynamic models for the operation of spiral-wound membranes in water treatment plants. Staying in porous media, Dixon and Madeiros [7] investigated radial dispersion in fixed-bed columns. They used computations to simulate the radial velocity and concentration profile for several different computer-generated tubes packed with spheres. The flow of air and methane was simulated, and the effects of the diameter of the packed column relative to the packing particle diameter were explored. It was found that the concentration exhibited a sharp decrease close to the column wall, resulting in a two- or three-parameter model being required to accurately predict this process, while models that assumed a single dispersion coefficient across the column were inadequate.

Two-phase flow phenomena in bench-scale bubble columns were studied in the work of Mohagheghian and Elbing [8]. Experiments were used to image the full distribution of air bubble diameters as air was injected into a water column, revealing the length scale of the most frequent bubble size. In addition, it was seen that the higher-order statistics of the bubble size distribution (i.e., skewness and kurtosis) affected the flow field in the column and could be used to indicate flow regime transitions. The wakes of bubbles in bubble-column reactors were studied by Ruttinger et al. [9], with emphasis on how these wakes were influenced by flow structures such as vortex streets. High-speed particle image velocimetry (PIV) was used to illuminate the flow structure in the wake of a single bubble. It was found that the vortex street interacted with the wake behind the bubble, enhancing transfer of momentum and mixing of mass. The paper of Cortes Garcia et al. [10] also focused on gas-liquid phases, but examined the determination of the gas-liquid mass transfer coefficient by critically examining Danckwert's method commonly used to determine this coefficient. It was found that, in cases where the mass transfer between the gas-liquid phases was intense, or in cases where a lot of liquid is present, there were significant errors in the calculated mass transfer coefficient. Cortes Garcie et al. [10] suggested the use of computations and experiments to fit the theoretical reaction and diffusion equations as best practice, while Mohagheghian and Elbing [8], Ruttinger et al. [9], and Cortes Garcia et al. [10] focused on adiabatic gas-liquid flow. Liao and Lucas [11] examined flash boiling flows, where the generation of bubbles was the result of a vaporization process as heat was added to the system. Available theories and correlations were evaluated with comparisons to computational fluid-dynamics simulation results. It was found that both conduction and convection were important for the bubble growth rate and that the existing correlations are applicable within specific ranges of the Jakob and Reynolds numbers.

Turbulent flow effects on mixing were examined by Nguyen and Papavassiliou [12]. Qualitative measures of mixing effectiveness and mixing quality were suggested, and Lagrangian computations and direct numerical simulations of turbulent flow allowed the evaluation of these measures for mixing particles with different Schmidt numbers. It was found that molecular diffusion and turbulence were both important in mixing when the turbulent flow was anisotropic. Dharmarathne et al. [13] also used direct numerical simulations of turbulent flow to investigate the formation of hot and cold spots when jets of fluid with lower temperature were injected into a channel flow. Details of the development of coherent structures and their role in the transfer of heat in anisotropic turbulence were documented. 
It was found that the coherent flow structures were modified by the injections, and these modifications were critical for the movement of hot fluid from the wall to the outer region. Engineering applications where cooling is necessary should, thus, ensure the generation of such coherent structures.

Finally, we thank the contributors to this special issue for sharing their research, and the reviewers for generously donating their time to select and improve the manuscripts.

Conflicts of Interest: The authors declare no conflict of interest.

\section{References}

1. Kanaris, A.; Mouza, A. Design of a Novel $\mu$-Mixer. Fluids 2018, 3, 10. [CrossRef]

2. Williams, C.; Kadri, O.; Voronov, R.; Sikavitsas, V. Time-Dependent Shear Stress Distributions during Extended Flow Perfusion Culture of Bone Tissue Engineered Constructs. Fluids 2018, 3, 25. [CrossRef]

3. Passos, A.; Tziafas, D.; Mouza, A.; Paras, S. Computational Modelling for Efficient Transdentinal Drug Delivery. Fluids 2018, 3, 4. [CrossRef]

4. Sobhansarbandi, S.; Maharjan, L.; Fahimi, B.; Hassanipour, F. Thermal Fluid Analysis of Cold Plasma Methane Reformer. Fluids 2018, 3, 31. [CrossRef]

5. $\quad$ Duong, H.; Xie, Z.; Wei, K.; Nian, N.; Tan, K.; Lim, H.; Li, A.; Chung, K.-S.; Lim, W. Thermal Jacket Design Using Cellulose Aerogels for Heat Insulation Application of Water Bottles. Fluids 2017, 2, 64. [CrossRef]

6. Koutsou, C.; Karabelas, A.; Kostoglou, M. Fluid Dynamics and Mass Transfer in Spacer-Filled Membrane Channels: Effect of Uniform Channel-Gap Reduction Due to Fouling. Fluids 2018, 3, 12. [CrossRef]

7. Dixon, A.G.; Medeiros, N.J. Computational Fluid Dynamics Simulations of Gas-Phase Radial Dispersion in Fixed Beds with Wall Effects. Fluids 2017, 2, 56. [CrossRef]

8. Mohagheghian, S.; Elbing, B. Characterization of Bubble Size Distributions within a Bubble Column. Fluids 2018, 3, 13. [CrossRef]

9. Rüttinger, S.; Hoffmann, M.; Schlüter, M. Experimental Analysis of a Bubble Wake Influenced by a Vortex Street. Fluids 2018, 3, 8. [CrossRef]

10. Cortes Garcia, G.; van Eeten, K.; de Beer, M.; Schouten, J.; van der Schaaf, J. On the Bias in the Danckwerts' Plot Method for the Determination of the Gas-Liquid Mass-Transfer Coefficient and Interfacial Area. Fluids 2018, 3, 18. [CrossRef]

11. Liao, Y.; Lucas, D. Evaluation of Interfacial Heat Transfer Models for Flashing Flow with Two-Fluid CFD. Fluids 2018, 3, 38. [CrossRef]

12. Nguyen, Q.; Papavassiliou, D. Quality Measures of Mixing in Turbulent Flow and Effects of Molecular Diffusivity. Fluids 2018, 3, 53. [CrossRef]

13. Dharmarathne, S.; Pulletikurthi, V.; Castillo, L. Coherent Vortical Structures and Their Relation to Hot/Cold Spots in a Thermal Turbulent Channel Flow. Fluids 2018, 3, 14. [CrossRef]

(C) 2018 by the authors. Licensee MDPI, Basel, Switzerland. This article is an open access article distributed under the terms and conditions of the Creative Commons Attribution (CC BY) license (http://creativecommons.org/licenses/by/4.0/). 